\title{
TEXT AND SUBTEXT IN DESIGNING HANDS-ON COURSE NOTES
}

\author{
CHELVA KANAGANAYAKAM \\ TRINITY COLLEGE
}

\section{ABSTRACT}

Hands-on computer courses often require carefully structured notes to complement the verbal instruction. They constitute an important area of technical writing, and the objective of this paper is to look at some of the underlying principles of designing course notes for hands-on courses.

With the need for and availability of computing technology for the non-technical person, the demand for focussed and user-oriented instruction has increased considerably. A combination of various factors, not the least of which is the formidable bulk of user manuals, has intensified the need for hands-on courses on very specific topics, such as "Managing Hard Disks", "Introduction to Dos", "Introduction to Electronic Mail", and so forth. In pedagogical terms, a hands-on course is similar to traditional classroom instruction, with the difference that each student gets a computer to work with and can expect a certain measure of individual attention from the instructor. Quite often, the student is given a set of notes which serves as a supplement to the instruction provided by the teacher. 
Hands-on course notes, like process descriptions, user manuals, on-line documentation, and step-by-step instructions, are task oriented, although they are distinguished by their special role in the pedagogical process. Although in generic terms they constitute a form of written instruction, course notes for a hands-on session are unique in that they are not intended to be stand-alone documents, like user manuals. What functions as a subtext for the notes is the commentary of the instructor who, in ideal circumstances, will ensure that there is no real conflict between the notes and the instruction. In some ways the combination of notes and commentary (or text and subtext) is ironic and paradoxical, because the registrant in a hands-on course usually is not comfortable working with the user manual. In that sense the hands-on document should be superior to a manual in its user-friendliness, and yet it does hot have to be so, because it has the support of the instructor, which the manual has to do without. The comparison does not presume that one is easier to design than the other; simply that they are different. The hands-on notes, then, presuppose interpretation by the instructor. It is a special kind of writing. The objective of this paper is to look at some of the underlying principles of this kind of writing, and some rhetorical and formatting aspects of designing course notes for hands-on sessions.

\section{OBJECTIVES OF NOTES}

In a general sense, course notes are documented instructions for a user. Specifically, the notes are intended to complement verbal instruction. There is, or should be, an orchestration between the narrative of the instructor and the notes. Also, the notes provide an uncomplicated step-by-step guide for the 
students to achieve certain results. Quite often, the students may not keep in touch with the material that is covered, and thereby forget some of the verbal instruction. In such a case, when they do return to the topic, the notes should enable them to recapture what was covered in the course. Also, since hands-on courses do not always aim to be comprehensive in their treatment of subject matter, the notes should function as a springboard for the students to read a more sophisticated manual and try out complex features. In order to design a set of notes that achieves these ends, the writer must comprehend the context of the classroom, which in fact is the primary testing ground for the document.

\section{UNDERSTANDING THE CONTEXT}

In order to be able to create an effective set of notes, one needs to understand the context in which it is primarily used. Despite the ostensible similarities, a hands-on environment is in many ways unlike a traditional classroom situation in that the instructor has less control over the students' activities, i.e., the actual handling of the computer. Experience indicates that the presence of a computer tempts students into digressing from the path mapped out by the instructor. Conformity is difficult to achieve, and often it pays not to insist on it, but to create a context that thrives on diversity. Some of the more common occurrences in a hands-on session are given below:

1. Students have varying typing skills, and unequal degrees of aptitude. A step that requires no more than a few minutes for one person could take much longer for another. What this leads to is a time gap in which students who have accomplished 
their task get bored and either look out of the window, work ahead of the class, or worse still, start trying out something new on the computer.

2. A related point is that it is not often possible to establish a rigorous time schedule for each section of the course, simply because the response of the students is often unpredictable. Usually, there is a need for the instructor to adjust her pace depending on the aptitude of the students. If what was planned as a fifteen minute exercise has to be done in seven minutes, the instructor will have to rely heavily on the notes to step up the pace, or, in some instances, request the students not to attempt the exercise in class, but to read the notes and attempt it at home. If this were to happen, then the instructor would simply explain the notes to the students who might include marginal notes to serve as pointers later.

3. While the instructor tries to address the whole class, individual students interrupt and demand attention. Unlike the situation in a classroom, this cannot be considered disruptive, for the student has a perfectly reasonable concern: if her problem is not resolved she will not be able to keep up with the rest of the class or follow subsequent steps.

4. While the instructor tries desperately to solve individual problems without holding up the entire class, eager beavers start going ahead on the basis of the notes. In this case, since they do not have the explanatory comments of the instructor, they may well get into unexpected problems. 
5. A hands-on course can hardly be achieved in a quiet atmosphere. The constant clicking of the keyboard, the hum of hard disks, the buzz of students asking each other for assistance or wanting to share their thoughts about the course or the instructor or both, not to mention the possibility of a printer in the background churning out pages of printed material, combine to create less than ideal acoustics. In this situation, the instructor, unless she has a stentorian voice, is likely to be inaudible to some. And the students involuntarily turn to the notes to seek guidance.

Hand-on course notes, then, are a special kind of communication, having similarities with other forms of instructions, but they require a different focus, and consequently a new approach to content, organization, layout and style.

\section{CONTENT}

Integrating Verbal and Written Communication

For the instructor who designs the course and the notes, the temptation is great to include a mass of material that is, in fact, redundant. Extraneous material often distracts the student who has to listen to the instructor, work on exercises, and look at the notes all at the same time. Hence instructions that are best given verbally need not be included. For instance, it may not be necessary to instruct the reader to boot up the computer or type a particular word in order to run a program. Particularly at the beginning, when new exercises are begun, the class is likely to be attentive. While it is difficult to be categorical about what is essential and what is not, 
it is important for the instructor to be aware of the potential pitfalls of including redundant material.

\section{Integrating Principle and Practice}

Hands-on courses are invariably very specific and task oriented. Hence there is a need to arrive at a judicious balance between theory and practice. Too much theory confuses the student, particularly if the concepts are not going to be translated immediately into practice. A very successful method is to begin with the conceptual basis of a task and then follow it with an exercise. Sometimes when certain theoretical concepts apply to all the exercises, it may be necessary to discuss them separately in order to avoid repetition at each stage. Typically, a paragraph or two of theory followed by an exercise that reinforces the theory is a method that students are often comfortable with. Figure 1, which explains to the student the process of inserting leader characters using Microsoft Word, shows an example of the modular approach that is often effective. 


\section{Leader Characters}

The space between entries in a table is a blank by default. You can fill this space with a dot, dash or underscore, all of which are called leader characters. Let us practise this.

1. Select rormat $\mathrm{Tab}$ set and type 3 in.

2. Move the arrow keys to the Leaders field and choose the dot by pressing the spacebar until the dot is highlighted.

3. Now press Enter.

Once you are back in the screen, press the tab key and you will see that as the cursor move from one spot to the other, the space in between will be filled with dots.

Figure 1. Principle and Practice

\section{Avoid Multiple Options}

User manuals, since they are reference sources, are obliged to include multiple ways of approaching a problem. Depending on circumstance and expertise, the user selects one which suits her best. For example, in MS Word, Format $T a b$ set provides access to the Tab menu, and so does Alt-ri. In hands-on course notes, providing multiple options is an invitation to chaos. The notes should draw attention to one method, and if she sees fit, the instructor could refer to other possibilities verbally. That way the more sophisticated student absorbs the additional material while the other stays content with what is provided in the notes. 
Escape Routes

When working with an unfamiliar program or keyboard, it is likely that some of the students will press something inadvertently and generate a totally unexpected response. It is usually difficult to anticipate all the possible disasters that students are capable of generating. User manuals are expected to have an elaborate troubleshooting section, but this is not so in the case of course notes. It is pointless to fill up pages explaining what should be done in the event of a disaster when several students are not likely to encounter any problems at all. Fixing problems individually is the task of the instructor or her roving assistant (if she has one), and, in any case, regardless of whether the document provides steps for recovery or not, the student is likely to call the instructor.

\section{Avoid Cross-Reference}

If steps have to be repeated, then it is best to repeat the steps rather than instruct the student to refer to a previous page or section. Given the time constraints and overall atmosphere of a hands-on course, cross-referencing is often ineffective.

It is equally important that if the students are involved in a complex, multi-step task they be given a sense of what the finished product will look like. In these instances, it is useful for the student to have the illustration always present on the table. Typically, course notes provide the illustration either at the beginning or at the end of the task. A far more productive approach is to provide the illustration on a detachable sheet of paper so that, even as the student turns the pages, the visual picture is always 
available. Figure 2 provides an illustration of a table that is best produced on a detachable sheet.

\begin{tabular}{|l|r|r|}
\hline \multicolumn{3}{|c|}{ Resources* } \\
\hline Tools & Cost & Weight \\
\hline Food & 100.00 & 23 \\
Rope & 2.50 & 1 \\
Stove & 60.00 & 5 \\
Tent & 45.00 & 10 \\
\hline \multicolumn{2}{|c|}{207.50} & 39 \\
\hline \multicolumn{2}{|c|}{} \\
*The data for the table was provided \\
by John Smith \\
\hline
\end{tabular}

Figure 2. A Complex Table

\section{Examples}

Attractive illustrations and examples are useful in manuals, for they emphasize the capability of a program or function. In a set of notes, they become a frustrating distraction or they tempt the student into branching off and attempting them on her own. If what is illustrated is not going to be achieved in class, then it is probably best not to include it.

\section{Appendices}

If background material must be documented, then the instructor has at least two options. One is to begin a new paragraph with "Note" in bold or bold italic and indicate to the student what the material is. If 
this is done, then the material should be brief, for it could distract the student from the more significant objectives of the course. A better alternative is to include it as an appendix for the student to read at her convenience. Here again, the appendix should not duplicate material that is available elsewhere.

\section{ORGANIZATION}

\section{Lists}

Exercises are usually mutli-step activities. If a task involves, say, five steps, it is best to list them sequentially rather than describe them in five paragraphs. Each step should not involve more than one instruction. And each step should be phrased carefully so as to eliminate ambiguity and preserve consistency within the document. Sometimes even the most trivial command like "Press ESC" needs to be repeated dozens of times in order to preserve consistency and also to make it easy for the student who is under considerable pressure. Even the most fundamental and obvious commands are often forgotten by students, and it certainly pays to err on the side of caution. It also helps to highlight keywords so that the experienced student does not have to read every word in the list of steps.

If the list exceeds ten steps, perhaps it would be best to create a break, review what has been done, and then proceed to a new list. Not only does this method prevent misreading, it also indicates to the student exactly what has been accomplished. Such a method enables the instructor to fix a problem quickly, when she is summoned by a frustrated and somewhat irate student. 
Mirroring the Screen

If the student is instructed to type in a body of text or some data, it may be useful to provide a visual illustration of what the screen should look like. In short, a correlation should be established between an illustration on the page and the appearance of the screen. Sometimes it pays to create a faithful copy of the screen using a screen-capture program. often it is not easy, or for that matter necessary, to provide a faithful copy of all the peripherals that appear on the screen. However, it is useful to include the material that needs to be typed verbatim in a box in order to signify a correlation between screen and text. Figure 3 is an illustration of material in a box that needs to be typed verbatim by the student.

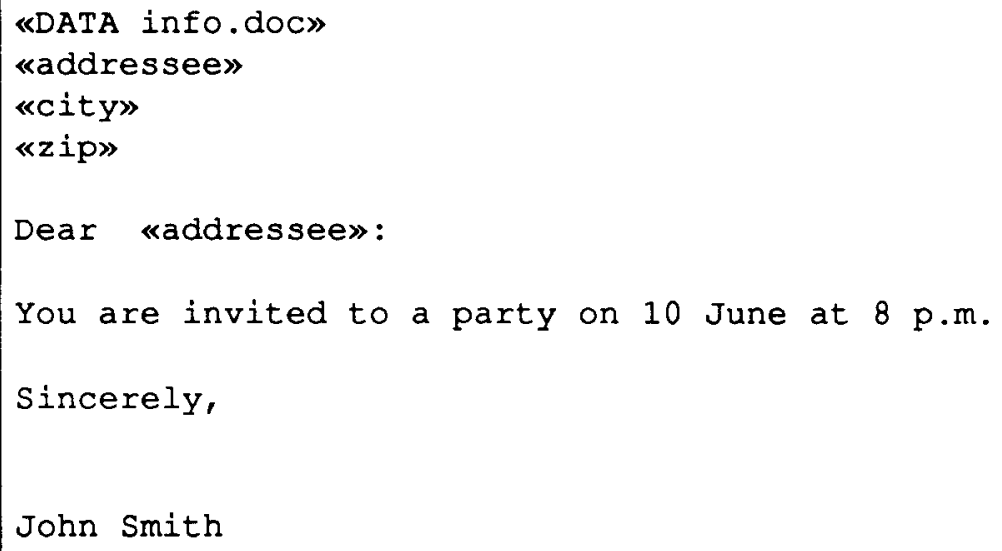

Figure 3. Typing Verbatim

While reproducing the screen in boxes is often reassuring to the student who feels pressured, it is equally important not to include boxes unless they 
serve an important purpose. Having too many leads the student to ignore them altogether.

\section{FORMAT}

Course notes, unlike user manuals, are usually not high-quality publications - they do not use glossy paper, color, or complicated graphics. However, formatting is crucial, simply because the student must read and comprehend the content in the midst of several other activities. Here again, it is difficult to be definitive, but some general guidelines may be observed in order to improve readability.

\section{Conventions}

A careful use of different fonts and stresses helps considerably in readability. For instance, major headings could be bold italic with a slightly bigger type size while subheadings could be simply bold. Similarly, material to be typed verbatim might be a different character set altogether. Similarly, if the student is directed to press certain keys on the keyboard, the references could be in bold, while secondary steps on sub menus could be in italics. Such a method provides consistency and enables the student to focus on what is essential.

\section{Spacing}

In a hands-on course it is not unusual for students to include a fair number of marginal comments based on the instructor's comments. Quite literally, writing between the lines is an important aspect of hands-on courses. Proper line spacing (again this would vary 
depending on the context) makes it convenient for the student. It is often helpful to leave a few blank lines at the end of every page for student notes.

\section{Widows and Orphans}

Both widows and orphans refer to important pieces of text, particularly headings and subheadings, appearing at the top or bottom of pages without any related text around them. In any form of writing, widows and orphans are awkward, but in hands-on course notes they are disruptive. In fact in course notes, if it is a choice between excessive amounts of white space and crowded text with inconvenient headings, the former is definitely the better choice.

\section{S TYIE}

Stylistically, hands-on course notes are not easily distinguishable from other forms of instruction. As in other forms of documentation, clarity and precision are significant, and any carelessness in the use of language tends to reflect badly on the subject matter and probably the instructor. Given below are a few features that probably need special consideration.

\section{Consistency of Labels}

Quite often, there is a conflict between the lexical items on the keyboards and the text. This is explained by the fact that not all keyboards are alike and locations of keys and names vary, and what is "Return" in one is "Enter" in another. Given this diversity, the instructor is tempted to do with her own experience and decide on a particular convention, 
thereby confusing the student who sees "Return" in the notes and is not aware that "Enter" performs the same function. A prudent course would be to check beforehand the conventions followed in the keyboards available to the student and follow them in the notes.

\section{Consistency of Commands}

A related source of ambiguity is the use of different phrases to describe the same command. From the instructor's point of view, the changes in syntax prevent repetition. For the user, the message becomes less clear. For instance, there is a difference between saying "Press EsC Format" in one instance and "Choose Esc Format" in another. The first implies a mechanical action while the second suggests a more conscious choice. Thus, even at the risk of repetition, it is probably best to opt for consistency.

Person

Instructions are usually in the second person. However, in a hands-on context, the instructor is usually more comfortable using "we" in the verbal instruction to create the impression of a collective endeavor. In the notes, the choice is more difficult because the activities, from the student's viewpoint, are both collective and individual. Hence, it is often useful to combine both the first and second person. When individual steps are described, perhaps "you" is appropriate, while during a review of what has been accomplished, "we" sounds more acceptable. For instance, a statement like "You should press Esc Format Border Box" followed by "Now we should see a rectangular box on the screen" strikes a balance between individual endeavor and collective accomplishment. 


\section{CONCLUSION}

The constant appearance of new products on the market, and the consequent difficulties in keeping abreast on the latest trends are going to increase the demand for hands-on courses. With this increase will come a greater diversity of audience. A more specialized audience is likely to be bored with a format that is more suitable for a novice. Hence definitive guidelines for designing notes cannot and perhaps need not be established. What is important to remember, however, is that hands-on course notes constitute an important and unique area of technical writing which has not been adequately explored and which, because of its crucial role as a supplement to verbal instruction, requires as much attention and care as any other instruction that has series implications for the reader.*

*I would like to thank Mr. Bob Bajwa of University Computing Services, University of British Columbia, for a critical and insightful reading of this paper.

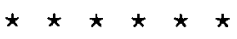

Formerly a Technical Writer/Programmer Analyst in the Computing Centre at the University of British Columbia, Chelva Kanaganayakam is now an Assistant Professor in the Department of English at Trinity College, University of Toronto. His areas of interest include Postcolonial literature, Technical writing and Victorian literature. 